\title{
SCAFFOLDING NOVICE INSTRUCTIONAL DESIGNERS' PROBLEM-SOLVING PROCESSES USING QUESTION PROMPTS IN A WEB-BASED LEARNING ENVIRONMENT
}

\author{
XUN GE \\ CHING-HUEI CHEN \\ KENDRICK A. DAVIS \\ The University of Oklahoma
}

\begin{abstract}
The present study investigated the effects of question prompts in scaffolding novice instructional designers solving ill-structured, instructional design problems in a Web-based learning environment. The effects of question prompts were studied under different prompting conditions (QuestionElaboration vs. Question-Guidance), taking into consideration various levels of learners' prior knowledge and experience. The study employed a comparative, multiple-case study design using the technique of think-aloud protocols, which were followed by interviews. Eight graduate students from the program of Instructional Design and Technology participated in the study. While the qualitative findings supported the previous research on the advantages of question prompts in scaffolding ill-structured problem solving, they also shed light on the specific cognitive and metacognitive functions, as well as limitations, of question prompts in different conditions. The study has implications for designing instructional scaffolds for supporting ill-structured problem solving of various domains in a Web-based learning environment.
\end{abstract}

\section{INTRODUCTION}

In recent years, educators and researchers have increasingly emphasized the importance of developing students' ability to reason with and apply knowledge to solve ill-structured problems in real world contexts (e.g., Cognition and Technology Group at Vanderbilt, 1990, 1993; Feltovich, Spiro, \& Coulson, 1989; 
Jonassen, 1999a; Koschmann, Kelson, Feltovich, \& Barrows, 1996). Ill-structured problems are those that we often encounter in everyday life. They are typically complex, ill-defined, and not well circumscribed; they either have multiple solutions or no solution at all (Chi \& Glaser, 1985; Sinnott, 1989; Voss \& Post, 1988). Solving ill-structured problems is much more complex and difficult than solving well-structured problems (Jonassen, 1997; Koschmann et al., 1996). Compared with well-structured problem solving, ill-structured problem solving has higher demands for cognitive and metacognitive abilities (Ge \& Land, 2004); thus it presents a greater challenge to many students who have difficulty applying knowledge to novel situations (Gick, 1986; Gick \& Holyoak, 1980; Feltovich, Spiro, Coulson, \& Feltovich, 1996), and who are weak in reflecting upon their performance and monitoring their learning process (Koschmann et al., 1996).

Due to the complexity of ill-structured problem-solving tasks and the difficulty experienced by novice learners in learning to solve ill-structured problems, simply engaging learners in ill-structured problem solving is insufficient, appropriate instructional scaffolds or support should be provided and embedded in the learning environment to guide learners during their work on complex, ill-structured learning tasks (van Merriënboer, Kirschner, \& Kester, 2003). Past research has examined a variety of scaffolding tools or techniques that support student learning (see Rosenshine \& Meister, 1992, for review); among them are question prompting (e.g., Scardamalia \& Bereiter, 1985; Scardamalia, Bereiter, \& Steinbach, 1984), expert modeling (e.g., Shoenfeld, 1985), reciprocal teaching (e.g., Palincsar \& Brown, 1984), and guided peer questioning (e.g., King, 1991, 1992, 1994).

Some researchers have specifically examined the use of question prompts in scaffolding student knowledge construction (King, 1992, 1994), integration (Davis \& Linn, 2000), and problem-solving processes in various content domains (King, 1991; Shoenfeld, 1985; Scardamalia et al., 1984). The overall findings have consistently pointed to the advantages of the use of question prompts in directing students' attention to important aspects of the problem, activating their schema, eliciting their explanations, and prompting them for self-monitoring and selfreflection (see Rosenshine, Meister, \& Chapman, 1996, for review). Based on the evidence of the positive results of self-monitoring prompts in his investigation, Kauffman (2004) argued that question prompts were a powerful instructional technique in promoting academic achievement, which could be automated in Web-based settings.

Despite the well-founded support for the use of question prompts to scaffold various learning tasks, few studies have examined the use of question prompts to support complex, ill-structured problem-solving tasks. Ge and Land (2004) conducted a critical analysis of question prompts in terms of their cognitive and metacognitive functions in supporting ill-structured problem-solving processes, which led to the theoretical assumption that question prompts could also be effective in supporting ill-structured problem solving. A couple of empirical 
studies have also examined the use of question prompts in scaffolding illstructured problem solving in the classroom setting. For instance, Greene and Land (2000) incorporated the technique of question prompts along with other scaffolding strategies to facilitate students of education major in completing a project on the use of instructional technology. Ge and Land (2003) provided a list of question prompts that were mapped to ill-structured problem-solving processes to guide undergraduate students in completing an ill-structured problem-solving task in information sciences and technology. While the studies revealed numerous advantages of question prompts in supporting ill-structured problem-solving processes, they also indicated some issues regarding question prompts as a scaffolding strategy. These issues will be addressed later in this article.

Hence, much has remained to be investigated and validated regarding the use of question prompts in scaffolding ill-structured problem-solving activities. This study is a continual effort to investigate the use of question prompts in facilitating students' ill-structured problem-solving performance, particularly their cognitive and metacognitive processes in representing problems, developing solutions, making justifications, and monitoring and evaluation, in an illstructured domain, such as instructional design.

\section{INSTRUCTIONAL DESIGN: ILL-STRUCTURED PROBLEMS IN AN ILL-STRUCTURED DOMAIN}

Instructional design can be characterized as a complex, ill-defined problemsolving process (Dick, Carey, \& Carey, 2001; Dijkstra, 2001; Jonassen, 1999b; Perez \& Emery, 1995; Reigeluth, 1999). Koschmann and his colleagues (1996) pointed out that the complexity and ill-structuredness of problems involves two aspects: a) the domain of knowledge itself, and b) the problems that challenge performance in that domain. Instructional design is one of such ill-structured knowledge domains in which the relationship between conceptual knowledge and the instance of knowledge application is irregular, and many different sets of concepts are necessary for understanding a case. Knowledge representation in instructional design may be configured and reconfigured to accommodate variance as it emerges because instructional design is a dynamic process, in which it is necessary to constantly monitor its environment to adjust to the changing conditions (Gustafson \& Branch, 2002).

Therefore, instructional design problems represent a special challenge to knowledge application in the domain. Like many other ill-structured problems, instructional design problems often do not have a well specified or clearly described situation, and the information needed to solve them is not entirely contained in the problem statement, which makes it less obvious what actions need to be taken in order to solve them (Chi \& Glaser, 1985). Because of the nature of ill-structured problems, there are no single, correct, or agreed-upon solutions; 
in other words, there may be multiple solutions, which requires the problem solver to select the best solution and consider various constraints and alternative courses of actions (Kitchener, 1983; Sinnott, 1989; Voss \& Post, 1988; Voss, Wolfe, Lawrence, \& Engle, 1991).

Instructional design involves higher-order thinking skills, such as analysis, interpretation, integration, and reflection (Dijkstra, 2001; Perez \& Emery, 1995). It requires instructional designers to be creative, active, and iterative in their design process (Gustafson \& Branch, 2002), and it also requires them to solve ill-structured problems in an ill-structured domain. In a study comparing expert and novice instructional designers, Perez and Emery (1995) found that an expert not only had far more complex knowledge structure and thinking than a novice, but also tended to select a design strategy, develop a plan, and systematically implement that plan to each successive design phase. An expert also executed more monitoring and evaluation skills throughout the design process and were more reflective in their selection and use of a design strategy. In addition, an expert spent more time on initial analysis and interpreting the design problem and considered a wide range of factors in combination with one another. The findings of this study was consistent with the previous research (e.g., Sinnott, 1989; Voss $\&$ Post, 1988) in that an expert problem solver frequently monitored and evaluated their problem-solving processes by comparing and selecting the best solution, considering various constraints and alternative courses of actions, and reasoning why he or she selects a particular problem space.

\section{TYPES OF QUESTION PROMPTS AND THEIR COGNITIVE AND METACOGNITIVE FUNCTIONS IN FACILITATING ILL-STRUCTURED PROBLEM-SOLVING PROCESSES}

Successful ill-structured problem solving requires cognitive (i.e., domainspecific and structured knowledge) and metacognitive knowledge (i.e., knowledge of cognition) and skills (i.e., planning, monitoring, and evaluation) (Chi \& Glaser, 1985; Kluwe \& Friedrichsen, 1985). A review of empirical studies reveals that question prompts can fulfill a number of cognitive and metacognitive functions in facilitating ill-structured problem-solving processes (Davis \& Linn, 2000; King, 1991, 1992, 1994; Lin \& Lehman, 1999; Scardamalia \& Bereiter, 1985).

Question prompts can be categorized into procedural, elaborative, and reflective prompts. Procedural prompts are characterized by directing students' efforts to complete a specific cognitive task, such as writing an essay or solving a problem. Scardamalia and her colleagues (1984) used question prompts such as "An example of this ....," and "Another reason that is good . . .," to facilitate learners in completing a writing task. King (1991) provided students with question prompts to guide them to ask each other questions that were 
designed to facilitate problem solving. Procedural prompts provide students a structure that leads them through the problem-solving processes.

Elaboration prompts were designed to activate schema and help students elaborate and articulate their reasoning process. Question prompts, such as "What does . . . mean?" and "What is the difference between . . . and . . . ?" were designed to help students make inferences and generalizations (e.g., King, 1992), whereas question prompts, such as "What is a new example of . . .?" and "Why is it important?" were intended to elicit students' explanations and directing their attention to understanding when, why, and how (e.g., Lin \& Lehman, 1999). Chi, Bassok, Lewis, Reimann, and Glaser (1989) found that the amount of working explanations generated by students were positively correlated with student learning success on problem-solving tasks. Empirical evidence (King, 1992; Lin \& Lehman, 1999) indicated that elaborative prompts can be used to support students' reasoning and justification process during ill-structured problem solving.

Reflective prompts, such as "To do a good job on this project, we need to ...," were intended to serve as cues to provoke students' reflections and elicit selfexplanations (Chi et al., 1989). Davis and Linn (2000) found that reflective prompts supported knowledge integration and encouraged reflection at a level that students did not generally consider. Reflection helps to make the connection between metacognitive knowledge and metacognitive control (Ertmer \& Newby, 1996). Recently, Kaufmann (2004) found that reflection prompts helped students to self-monitor and study strategically. Depending on the context, the elaborative prompts can also be used to fulfill the function of reflective prompts. Therefore, it is expected that reflection prompts may play an important role in helping students to self-monitor their problem-solving processes and consider various perspectives and values regarding their selected solutions.

Using a combination of different types of question prompts (procedural, elaborative, and reflective) to scaffold undergraduate students' ill-structured problem solving, Ge and Land (2003) found that the students (in both individual and group conditions) who received question prompts during problem solving performed significantly better than those who did not receive question prompts in the processes of problem representation, developing solutions, making justification, and monitoring and evaluation. In addition, the qualitative data suggested a number of advantages of question prompts in facilitating students' reasoning and problem-solving processes, for example, prompting students to make intentional efforts to identify relevant factors, constraints and information during problem representation process; helping them to organize information and plan for the solution process; facilitating students to articulate their solution process and construct sound arguments; evaluating the selected solutions, and comparing alternatives and justifying for the most viable solutions. 


\section{PURPOSE OF THE STUDY}

In their investigation, Ge and Land (2003) also noted some issues regarding question prompts as a scaffolding strategy; for instance, some students ignored the question prompts that were provided to them, resulting in a lack of attention to some important aspects of the problem. Similarly, Greene and Land (2000) found that question prompting was insufficient as a scaffold because students sometimes omitted questions or answered them superficially, thereby failing to engage in deeper processing. Those issues led us to ask the question: Would question prompts be more effective if they were designed in a way that learners were "forced" to follow the procedure by responding to each of the questions (Zellermayer, Salomon, Globerson, \& Givon, 1991)? Zellermayer and her colleagues (1991) found that the unsolicited group, who had to receive continuous metacognitive-like guides, showed the evidence of having internalized the explicitly provided guidance by writing better essays than the control group and the solicited guidance group, who received the same metacognitive-like guides as the unsolicited group but only upon their voluntary solicitation.

On the other hand, Davis and Linn (2000) found that although prompts were useful in helping students structure and complete an activity, they also tended to reinforce following "step by step" procedures rather than reflecting and connecting ideas. In this case, we wondered if a list of question prompts presented as guidelines would help learners see an overall picture and help them to connect ideas holistically for self-reflection. Therefore, we were in a dilemma that we would structure and reinforce step-by-step procedural guidance and yet we were confronted with the possibility that students might overlook a larger picture of the problem-solving processes. This paradox led us to ponder upon the following questions: What would be an appropriate context for question prompts to work effectively? And what would be an effective way to present question prompts (e.g., question prompts presented as guidelines as opposed to question prompts requiring responses), given a specific learning context?

King (1992) argued that the effective use of question prompts relied on students' prior knowledge, which played an important role in elaborated learning. If students do not have relevant prior knowledge and experience, they may be less likely to be activated by and benefit from question prompts. Therefore, it is necessary to extend our understanding of the use of question prompts as a scaffold by investigating conditions in which question prompts work effectively, with the consideration of students' prior knowledge and experience on a given domain as well as other dynamic components that may affect students' intentional use of question prompts. The present study will contribute to the recent research efforts on adaptive scaffolding (e.g., Azevedo, Cromley, \& Siebert, 2004; van den Boom, Paas, van Merriënboer, \& van Gog, 2004), yet with a distinctive difference that this study addresses a learning context 
in which question prompts are provided when dynamic, human feedback is not immediately available, as in a Web-based learning environment.

The purpose of this study is twofold: a) to validate the results of the previous research on the effects of question prompts in scaffolding ill-structured problemsolving processes in an ill-structured domain and in Web-based learning environment; b) to investigate conditions in which question prompts work effectively to support ill-structured problem-solving processes; and c) to examine the relationships between different question prompting conditions and different levels of prior knowledge and experience. The research questions were formulated as follows:

1. Do question prompts have cognitive and metacognitive effects on students' problem-solving processes in the domain of instructional design in the Web-based learning environment? If so, how do they influence those processes?

2. In what contexts do question prompts work most effectively in facilitating students' problem-solving processes in the domain of instructional design?

3. How do question prompts presented in different prompting conditions (questions requiring responses vs. questions presented as guidelines) interact with different levels of learners' prior knowledge and experience in their problem-solving processes?

\section{METHOD}

\section{Research Design}

This study aimed to seek an in-depth understanding of students' reasoning and problem-solving processes in instructional design when supported by question prompts. A qualitative study method with multiple-case comparisons was applied for the investigation (Stake, 2000; Yin, 2002). Each individual participant was considered a case, which was compared with other cases within the same condition and in other conditions, such as Question-Elaboration (QE), Question-Guidance (QG), or No-Question-Prompts (NQ) condition. The QE condition refers to the condition in which students were required to respond to the question prompts as opposed to the QG condition, in which the question prompts were presented as guidelines.

Thinking aloud was used as a major technique to gather data about participants' reasoning processes during problem solving. It is a technique that has been commonly used by researchers to directly capture, observe, and examine individuals' reasoning processes during problem solving (e.g., Voss \& Post, 1988; Sinnott, 1989). Additional data sources consisted of the participants' solution reports and follow-up interviews. The solution reports served to provide additional information about participants' cognitive and metacognitive processes 
during problem solving while the follow-up interviews were intended to explore participants' perceptions of the problem-solving processes and the effects of question prompts on those processes.

\section{Participants}

The participants were eight graduate students (four female and four male) in the program of Instructional Psychology and Technology (IPT) at a major university in the southwest of the United States. The participants were from diverse ethnic backgrounds, representing different levels of prior knowledge and experience in instructional design, length of time they had studied, and the number of courses they had completed in the graduate program. All but one participant had taken at least one core course in Instructional Design (ID). One participant was taking his first ID course when the study was conducted. A pre-assessment was administered to measure the participants' declarative knowledge on instructional design, particularly needs assessment, prior to the study. Table 1 presents the profiles of the eight participants.

\section{Question Prompt Conditions}

The eight participants were assigned to one of the three study conditions ( $Q E, Q G$, and $N Q)$ in the Web-based learning environment. In the $\mathrm{QE}$ condition, the participants were required to write responses to each of the question prompts in the text boxes provided on the Web page. in the QG condition, the participants were provided with the same list of question prompts, but they were not required to write written responses; no space was provided to record their responses on the Web page. Instead, they were instructed to use the question prompts as a guide for their problem-solving task. The NQ condition was designed to observe how students would approach the same problem-solving tasks without the cognitive and metacognitive support of the question prompts. Hence, the NQ condition served as a reference point for comparison with the other two conditions.

The participants with similar levels of prior domain knowledge, relevant background experience, and the length of study in the graduate program were assigned to different conditions so that the three conditions were comparable. However, we were not able to get a sufficient number of participants so that the assignment ended up with four participants in the QE condition, three in the QG condition, and one in the NQ condition. Since every participant had to solve a problem for a pre-case study, we could observe how the participants in the QE and QG conditions approached the problem-solving tasks when question prompts were not provided. This source of data would remedy the situation when there was a lack of participants in the NQ condition. 


\section{Table 1. Participants' Profiles}

\begin{tabular}{|c|c|c|c|}
\hline Participant & $\begin{array}{l}\text { Question } \\
\text { prompt } \\
\text { format }\end{array}$ & $\begin{array}{l}\text { Prior knowledge } \\
\text { and experience } \\
\text { relevant to ID }\end{array}$ & $\begin{array}{l}\text { Length in the } \\
\text { graduate program }\end{array}$ \\
\hline Tina & QE & $\begin{array}{l}\text { Experienced high school } \\
\text { teacher in social studies; } \\
\text { have designed school } \\
\text { curricula. }\end{array}$ & $\begin{array}{l}\text { Have completed the first years' } \\
\text { coursework; have taken one ID } \\
\text { course and other courses in } \\
\text { educational psychology. }\end{array}$ \\
\hline Simon & QE & $\begin{array}{l}\text { Experienced high school } \\
\text { science teacher; have } \\
\text { designed school curricula. }\end{array}$ & $\begin{array}{l}\text { New student in the IPT program; } \\
\text { in the process of taking the first } \\
\text { ID course. }\end{array}$ \\
\hline Jane & QE & Little work experience. & $\begin{array}{l}\text { Have completed the first years' } \\
\text { coursework; have taken two ID } \\
\text { courses and a few Web and } \\
\text { multimedia instructional design } \\
\text { courses }\end{array}$ \\
\hline Elaine & QE & $\begin{array}{l}\text { Teaching Assistant; have } \\
\text { training experience in } \\
\text { professional settings. }\end{array}$ & $\begin{array}{l}\text { Have taken two ID courses; on } \\
\text { the point of completing the } \\
\text { graduate program and } \\
\text { graduating. }\end{array}$ \\
\hline Kathryn & QG & $\begin{array}{l}\text { Experienced middle school } \\
\text { science teacher; have been } \\
\text { working as a graduate } \\
\text { assistant designing online } \\
\text { courses. }\end{array}$ & $\begin{array}{l}\text { Have taken two ID courses and } \\
\text { a number of Web and } \\
\text { multimedia design courses; on } \\
\text { the point of completing the } \\
\text { graduate program and } \\
\text { graduating. }\end{array}$ \\
\hline Chris & QG & $\begin{array}{l}\text { Instructional technologist in a } \\
\text { school district; have designed } \\
\text { and implemented training } \\
\text { workshops for in-service } \\
\text { teachers' professional } \\
\text { development. }\end{array}$ & $\begin{array}{l}\text { Have completed a number of } \\
\text { courses over several semesters, } \\
\text { including two ID courses and } \\
\text { some Web and multimedia } \\
\text { instructional design courses. }\end{array}$ \\
\hline Steven & QG & $\begin{array}{l}\text { Little prior professional work } \\
\text { experience; have worked as } \\
\text { an intern for a short period, } \\
\text { designing Web-based } \\
\text { training materials at a company. }\end{array}$ & $\begin{array}{l}\text { Have completed his first year } \\
\text { study, including two ID courses; } \\
\text { strong in multimedia } \\
\text { development. }\end{array}$ \\
\hline Shawn & $N Q$ & Little work experience. & $\begin{array}{l}\text { Have completed the first years' } \\
\text { coursework; have taken two ID } \\
\text { courses and a couple of multi- } \\
\text { media development courses. }\end{array}$ \\
\hline
\end{tabular}




\section{Contexts, Tasks, and Question Prompts}

A Web-based learning environment was developed for this study, including a database designed for students to submit, store, and retrieve data they have entered. The Website started with an overall description of the site, the purpose, and the tasks the students would be working on. Then the pre-case study was presented as a pre-assessment of the participants' problem-solving performance before receiving the intervention of question prompts. The pre-case study presented an authentic instructional design scenario, in which a problem associated with a graduate hypermedia course was described. The case study called for participants' help in conducting a needs assessment aiming at improving the quality of the instruction and to satisfy students' needs. The participants were required to analyze the problem and suggest a plan for conducting needs analysis by applying their instructional design knowledge and skills. A text box was provided for students to record their analysis and type their solution report, which was to be submitted upon completion.

Next, another two authentic case studies (Case Study 1 and Case Study 2), which were similar to the pre-case study in structure, content, and tasks, were presented sequentially. Appendix A shows Case Study 1 and its task description. The participant in the NQ condition was asked to complete each of the two case studies by producing a final solution report (i.e., a needs assessment plan) while no question prompts were provided. Whereas, the participants in QE and QG conditions were provided with a list of question prompts after the presentation of the case study. The participants in the $\mathrm{QE}$ condition were required to respond to each of the question prompts by typing their responses in the text boxes provided. Their completed responses would then be submitted, saved to the database, and displayed on the next screen, where the participants could copy and paste their prior responses, which then would be organized and edited into final solution reports. The participants in the QG condition were simply asked to think about the question prompts and used them to guide their problem-solving processes and develop a solution report. Figure 1 illustrates the three conditions respectively.

The case studies were generated from some real-world design projects, sharing a surface structure embedded with the same underlying instructional design principles. The first author served as a subject matter expert in designing the case studies and the question prompts. The question prompts (refer to Appendix B) were intended not only to provide novice instructional designers step-by-step procedural guidance in completing the case studies, but also to prompt them to elaborate their thinking, articulate their thoughts, make justifications for their decisions and solutions, and monitor and evaluate their reasoning and problemsolving processes. Here are some examples of the question prompts: "Is this design project in response to a problem or a need? ... How do you know? How can you determine it?" "What kind of investigation techniques are you going to use? Why do you suggest using each of the techniques above?" As shown by 


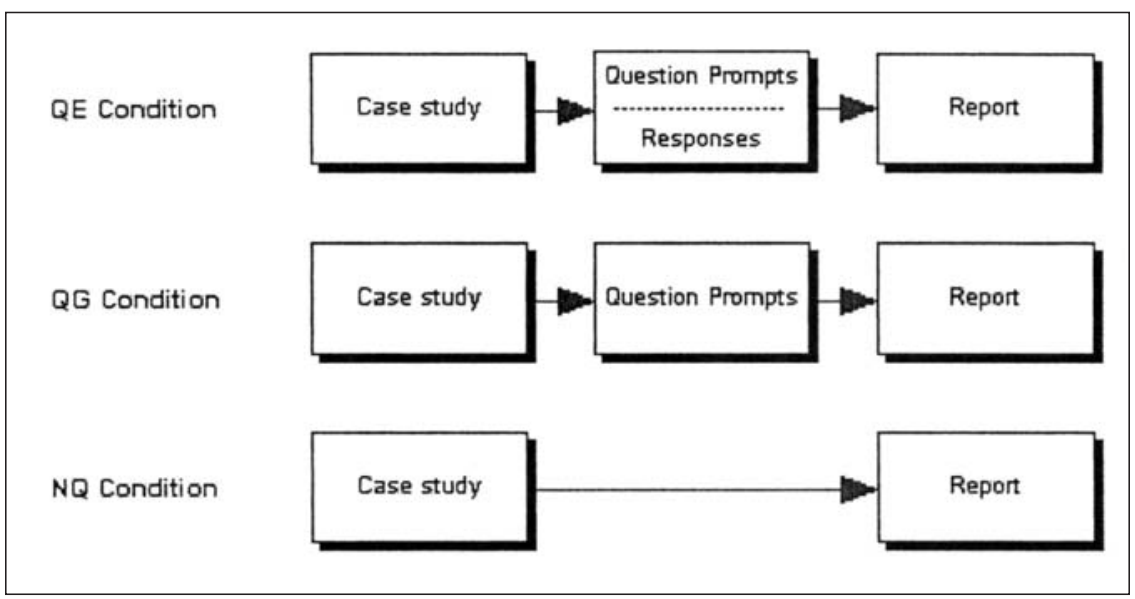

Figure 1. Different treatments for Question-Elaboration (QE) Condition, Question-Guidance (QG) Condition, and No-Question Prompt (NQ) Condition.

the examples, these question prompts also served the purposes of elaborative prompts and reflective prompts.

\section{Procedure}

The study sessions were administered to different participants at different times. Before a study session began, the researcher would explain and demonstrate to the participant the think-aloud procedure. Not until a participant understood how to talk aloud was he/she asked to start verbalizing his/her thinking process while working on the problem-solving task. All the think-aloud protocols were recorded using a digital audio recorder.

The study procedures consisted of two sessions: a) the case-study session and b) the interview session. The first session consisted of a pre-case study and two case-studies while the think-aloud was performed simultaneously as the participant was working through the problem-solving tasks, with or without the support of the question prompts. Every condition had a different login account and password, which directed the participants to a Web site of a designated prompting condition. After logging into the Web-supported learning system, the participants were asked to work on the pre-case study, followed by Case Study 1 and Case Study 2 . The participants took approximately three hours to complete the casestudy session, but the length of time varied from individual to individual. The first two authors were present during the think-aloud process, observing, taking notes, and audio-recording. Occasionally, they reminded the participants to continue to talk aloud or to speak louder. 
The interview session was administered about a week later. Each interview took approximately 20 minutes. Structured questions were asked of the participants about their experience during the problem-solving processes, particularly their problem-solving approaches and strategies. The participants in the QE and QG conditions were asked of the effects of the question prompts on their problem-solving processes.

\section{Data Sources and Analysis}

The primary data sources were composed of the think-aloud protocols, the solution reports on needs assessment plans, and the follow-up interviews. Miles and Huberman's (1994) data analysis model, which consisted of data reduction, data display, and conclusion drawing, was used to guide the qualitative data analysis of the multiple-case studies. Data analysis was carried out at three levels. The first level involved coding and labeling of the think-aloud protocols. The second level included organizing the reduced data generated at the first level and displaying them in matrixes for the purpose of within-case and cross-case comparisons. At the third level, patterns were identified and themes were generalized based on the prior two levels of analysis.

The three authors first coded the think-aloud protocols independently, and then they met to discuss their initial interpretations. Next, they used Excel to create a matrix to display data for within- and cross-case comparisons. The matrix, which showed the participants' number, pseudonyms, and the study condition they were assigned to, was set up in a way that comparisons could be made within and across cases of different conditions. Specifically, the matrix also visually displayed the problem-solving processes in problem representation, developing solutions, making justification, and monitoring and evaluation. Each of the problem-solving processes had a column for "Indication" and a column for "Examples" so that the authors could fill in the cells with the information from the think-aloud protocols, which were later triangulated with other sources of data, including solution reports and interviews. The interview data, which were transcribed and analyzed using the same data analysis approach as used for the think-aloud protocols, were used to supplement, verify, and support findings from the think-aloud protocols. The three authors compared notes, verified codings, and discussed interpretations through numerous meetings in order to reach a consensus.

The a priori approach (Miles \& Huberman, 1994) was used to analyze the solution reports qualitatively in four areas: problem representation, developing solutions (i.e., needs assessment plan), making justifications, and monitoring and evaluation. Problem representation was concerned with determining the need, analyzing different people and different perspectives, and analyzing external resources. Developing a needs assessment plan involved generating hypothesis, determining data types, sources, and investigation methods. Constructing 
argument and presenting rational involved creating clear links between problem representation and possible solutions. Similarly, the authors first analyzed and scored the participants' reports for all the case studies independently, then they met to compare notes, resolve differences, and reach an agreement on the scores. The data of different sources were finally triangulated to generate themes to provide answers to the research questions.

\section{FINDINGS}

\section{Research Question 1}

\section{Overall Findings}

The overall findings indicated that question prompts had positive effects in facilitating students' cognitive and metacognitive thinking processes when solving ill-structured instructional design problems, even though for some participants the written solution reports did not completely reflect the level of reasoning as demonstrated in the think-aloud process. The major findings from the think-aloud data are summarized below.

Tina $(\mathrm{QE})$ and Elaine $(\mathrm{QE})$, who were already high performers in the pre-case study, performed better in the two case studies than the pre-case study, especially in elaborating their thoughts for problem representation, constructing argument, monitoring the process, and assessing the alternatives. For Simon (QE), who was new to the field of Instructional Design, and for Jane (QE), who had very little relevant real-world experience in instructional design, the question prompts showed positive effects in guiding them through the problem-solving processes in the subsequent case studies. Kathryn (QG) did not seem to know how to approach the problem during the pre-case study, but she made observable progress in the subsequent two case studies, especially in ways she organized her thoughts and monitored her problem-solving processes. For Chris (QG), it was shown that the question prompts were beneficial in prompting him to elaborate his thinking, make justifications, and monitor his problem-solving processes.

In contrast, Shawn (NQ), who did not receive any question prompts, showed consistent patterns in solving problems across the three case studies: spending little time representing the problem, and almost immediately jumping to solutions by listing what he was going to do in response to the case studies.

The results of the solution report analysis indicated some inconsistencies with the findings from the think-aloud protocols. Some participants made observable progress in Case Study 1 and 2, some performed consistently well in all the three case studies, while the others demonstrated little improvement in the three written reports. For example, in comparison with the pre-case study, Kathryn made obviously greater progress in Case Study 1 and 2. She scored higher in every problem-solving process, including problem representation, developing solutions, and making justifications. Chris scored higher in the process of making 
justifications for Case Study 1 and Case Study 2 than for his pre-case study report while Simon had better scores in developing solutions and making justifications for Case Study 1 than for the pre-case study. Unfortunately, Simon became tired during Case Study 2 and did not complete it.

Tina's solution reports were scored consistently high across the three case studies. Elaine was also one of the high performers for the pre-case study, but her reports for Case Study 1 and 2 were scored higher than her report for the pre-case study in the process of problem representation. However, by examining the solution reports and scores alone, Jane and Steven showed little sign of improvement across the three case studies.

The examples from the think-aloud protocols presented below demonstrated that the written solution reports did not fully capture or record the participants' reasoning processes, which were, in fact, influenced and facilitated by the question prompts in many ways, particularly in representing problems, providing explanations, making justifications, monitoring and evaluating their problem-solving processes.

\section{Facilitating Problem Representation}

In the pre-case study, Simon generated some hypotheses for the causes of the problem, but he failed to offer explanations and develop a solution. When working on Case Study 1, Simon was observably prompted by the questions to engage in deeper cognitive processes of determining the need for developing a Web-based instruction. He was also observed engaging in analyzing the causes for "students" low motivation in taking the literature course." In addition, Simon also elaborated and compared alternative causes of the problems, differentiating "the instructor's anticipation" from the "real needs of the students."

Although her solution report scores showed little improvement in Case Study 1 and 2, Jane actually became more elaborative in the process of problem representation, as observed from the think-aloud protocols. During the pre-case study, Jane assumed that there was a need to develop the Web-based instruction, which prevented her from further analyzing problem causes and identifying relevant factors. In Case Study 1, on the contrary, Jane was able to engage in the process of identifying constraints and exploring alternatives that might attribute to students' low motivation in taking the literature course.

Kathryn had a hard time representing the problem for needs analysis presented by the pre-case study. She tried to activate her schema by reading the case description repeatedly, but she still failed to provide any answers or solutions to the problem. When working on Case Study 1 and Case Study 2, however, Kathryn was observed to be seeking alternatives such as "if . . ., then ...," generating hypothesis, identifying relevant factors and contexts, such as "learning environment, learner characteristics, and feelings." The question prompts apparently facilitated Kathryn to elaborate her thoughts and analyze the problem. 


\section{Prompting for Developing a Needs Assessment Plan}

In the pre-case study, Simon had failed to generate a needs-assessment plan. However, in developing solutions for Case Study 1, he responded to the question prompts and suggested the techniques he would use to conduct the needs assessment and the people he would like to investigate. He backed up his solutions

by a rationale. Kathryn failed to suggest a needs assessment plan for the pre-case study. However, she was able to suggest alternative methods for needs assessment for both Case Study 1 and Case Study 2. In the same way, Elaine was able to consider alternatives and using "if . . ., then ..." approach in the process of selecting a solution in the latter two case studies; whereas she only suggested a single solution for needs assessment for the pre-case study.

\section{Prompting for Making Justifications}

For Chris, the most obvious effects of question prompts were elaboration and justification, which was evident in Chris' think-aloud protocols. Chris tended to be brief in his thinking and writing. He tended to leave a gap in his logical reasoning for others to infer what he was trying to say. However, when working on Case Study 1 and Case Study 2, Chris was observed elaborating on the needs assessment techniques he was going to use and explaining why he chose to use those techniques.

In the pre-case study, Steven did not identify the cause of the problem, but in Case Study 2, he was engaged in the process of identifying the causes of the problem, explaining what he thought might be the cause of the problem. He was also seen justifying the needs for "developing an EPSS system" in response to Case Study 2.

Simon exhibited a logical chain in the process of problem representation. He was questioning the "needs for Web-based instruction" rather than assuming that there was a need as he did in the pre-case study. For example, he articulated that "WBI as a solution depends on the needs ..." and he demonstrated a logical reasoning, such as "If . . ., then ..." argument in his problem representation process. Similarly, Kathryn, Elaine, and Jane were also observed making efforts in creating logical links when articulating their thoughts in representing the problem.

\section{Facilitating Monitoring and Evaluation}

It was evident that Chris was aware of the purpose of the question prompts. He used the question prompts as guidance. This was evident by going back to read the question prompts and later referring back to the problem several times to monitor his thinking process. Kathryn was also observed going back and forth between the question prompts and the problem-solving tasks throughout the problem-solving processes in Case Study 1 and Case Study 2. She used the 
questions to guide her through the problem-solving processes, such as problem representation, developing solutions, and making justifications.

Prompted by the question prompts, Tina intentionally made efforts to evaluate the validity of the "conceptual information" during problem representation stage. The data also showed that the question prompts helped Elaine to assess alternative solutions.

\section{Serving as a Procedural Guidance}

The interview data not only confirmed the findings from the think-aloud protocols, but also provided additional insights into the effects of the question prompts. For example, both Tina and Elaine suggested that the question prompts had helped them organize information and break down the problem-solving tasks into manageable steps. Both Jane and Simon mentioned that the question prompts had helped them to focus their attention on the most important and relevant information. Simon added that the question prompts also helped him to activate his prior knowledge, elaborate his thoughts, and reflect on the problem-solving processes.

To summarize, the question prompts had positive effects on their problemsolving processes. In problem representation, question prompts helped the participants to elaborate their thinking, define the problem, and identify constraints and causes of the problem. In particular, the question prompts had enabled the participants to explore alternative possibilities in problem representation, which was obviously lacking in the pre-case study. The ability in seeking alternative causes further led the participants to compare and consider alternative solutions. In addition, the question prompts facilitated the participants to articulate their reasoning process and make justifications for problem representation and solutions. Above all, the question prompts served as a procedural scaffold in guiding the participants to generate solutions, explain their rationales for the solutions, monitor their problem-solving processes, and facilitate them to evaluate alternative solutions.

\section{Research Question 2}

The qualitative analysis also pointed to some limitations of the question prompts. First, question prompts required relevant prior knowledge and sufficient schema in order to be effective. If there was little prior knowledge or experience to relate to or if one's schema was narrow and limited, question prompts could hardly activate schema to elaborate thinking. Second, if students had already some pre-made assumptions or bias toward an issue, they might simply overlook some relevant question prompts but rather build their argument based on their own assumptions. In this context, the question prompts might not be able to function effectively. Third, while the question prompts worked well for most of the participants, they might not be as effective for advanced performers as for novice 
learners because the question prompts may have interfered with their thought flow during their problem-solving processes. Fourth, while the question prompts might be effective in facilitating the participants' reasoning process in problem solving, they might not necessarily be effective in facilitating the productive process, such as writing a solution report.

In other words, question prompts worked most effectively in the following contexts: when students had sufficient schemata about the content domain, when they were free of pre-assumptions, when they had lower competence level in problem solving, and when they were used to facilitate cognition and metacognition instead of production, such as writing a solution report. The limitations of the question prompts were illustrated with examples below.

\section{Lack of Prior Knowledge and Relevant Schema}

It was observed that when Steven was working on Case Study 1, he kept saying "I don't know ...." in response to the list of the question prompts. He eventually gave up his efforts in answering those questions. He said "It was hard . .." in the interview. Whether it was due to the failure to recall situations that helped him to answer the questions or a lack of knowledge to answer the questions, the question prompts had not been able to help Steven to activate his schema. However, Steven performed better in the second case study, when he was able to respond to the question prompts because he could relate the case study to his prior experience.

Even though question prompts had helped Jane to identify relevant factors and constraints and consider alternative causes in Case Study 1, her problem space was strictly confined to a couple of factors, such as "cost and resources," which really limited her solution selections for the needs assessment plan. She was observed following the similar pattern when working on Case Study 2. In Jane's situation, due to her limited prior knowledge and problem space, the question prompts had not helped to expand her schema to include additional factors or constraints to consider.

\section{Making Assumptions}

In Case Study 1, although the question prompts asked the participants to determine the need and justify for it before thinking about seeking solutions in designing Web-based instruction, some participants still assumed that the Web-based instruction was needed in the first place and proceeded with the needs analysis subsequently. This was shown by the cases of Jane and Tina, who had already determined at the beginning that the Web-based instruction was necessary for the situation as described by Case Study 1 without conducting any needs analysis. 


\section{Interference with Thought Flow}

Like Tina, Elaine used the question prompts as a checklist to monitor her problem-solving processes. The think-aloud protocols showed that Elaine was more elaborative when working on Case Study 1 and 2 than on the pre-case study. However, Elaine reported in the interview that the question prompts (to which she had to respond one by one) had interfered with her thought flow because she already knew what to do and how to do it, and she probably did not need much scaffolding. It may be inferred that question prompts worked more effectively for learners with lower competence in problem solving.

Incompatibility between the Reasoning Process

and the Written Report

To further investigate the participants' ill-structured problem-solving performance, a comparison was made between the participants' solution reports and their reasoning process as transcribed in the think-aloud protocols. Not all the think-aloud protocols were compatible with the written reports. For example, in Chris' case, although his two problem-solving reports had improved in rating compared with that of the pre-case study, its quality remained incompatible with his think-aloud process, which showed the evidence of a significant improvement. Jane also failed to organize the information well in writing, although her thinkaloud protocols and her responses to the question prompts were much more elaborative. These cases indicated that writing and thought elaboration were two different cognitive tasks, which were not necessarily compatible to one another.

\section{Research Question 3}

\section{The Effects of Question-Elaboration vs. Question-Guidance Prompts}

It is found that the question prompts in the QE condition elicited more elaborations than the QG condition, whereas the question prompts in the QG condition facilitated the participants in monitoring their problem-solving processes more than in the QE condition. Moreover, we also found that the elaborative feature of the QE condition and the monitoring feature of the QG condition interacted with the participants' prior knowledge and experience. Reported below were a few cases and examples.

The QE questions "forced" the participants to elaborate their thinking, which were beneficial in helping them to define the problems or "the needs," identify the causes, and provide rationale for their decisions or solutions, as shown in the cases of Simon and Jane discussed earlier. However, as reported by Elaine, being "forced" to respond to each of the specific questions interfered with her thinking process because she believed that she had already acquired the competence to perform this type of task. Interestingly, Tina, who was identified as another 
advanced performer, did not complain of being "forced" to respond to each of the question prompts in the interview. Instead, she thought that the question prompts were helpful guidelines. In her case, a list of QG questions might have served her purpose equally well or even more efficiently.

Because of the way the QG question prompts were presented, students could simply skip the prompts without using them. For instance, Chris skipped the questions that he thought were unimportant and chose to respond to those he regarded as useful. In this circumstance, the question prompts mainly fulfilled the function of monitoring problem-solving processes. Presumably, it required the participants' ability to determine which questions were useful and which ones could be skipped. In addition, the QG questions might allow some students to give up their efforts when working through a difficult problem, as it was shown in Steven's case. It was observed that Steven went through the question list but finally gave up answering those questions, either because he did not know how to answer them, or as he said later in the interview, he did not realize that he had to answer those questions. We wondered if it would make a difference to his reasoning and problem-solving performance if Steven was "forced" to respond to the questions, just as in the QE condition.

Kathryn's case seemed to suggest that different prompting conditions did not matter much because she used the QG question prompts as if she was using the QE questions. She was seen going through and responding to each of the questions; prompting herself to consider various aspects of the problem and construct sound arguments regarding an instructional need. At the same time, she also used the question prompts to monitor her problem-solving processes. This case seemed to suggest that QG questions could work just as well as QE questions if students perceived them as beneficial and deliberately used them. The successful use of the question prompts seemed to depend on the intentional use of learners.

\section{The Effect of Real-World Experience on} the Use of Question Prompts

The pre-assessment of the participants' basic knowledge about instructional design, which preceded the pre-case study, suggested that one's declarative knowledge was not an accurate indicator of students' problem-solving experience. For example, both Kathryn and Simon performed well in the pre-assessment, but their performance in the pre-case study did not appear compatible with their pre-assessment results. There seemed to be a gap between what learners knew and what they actually did. There was no apparent connection between the participants' declarative knowledge and their problem-solving performance, as well as their effective use of question prompts.

The data seemed to signify that real-world knowledge and experience played an important role in the successful use of question prompts to facilitate problem-solving processes. The multiple-case analyses, with the references to the 
participant's profiles indicated that the question prompts worked most effectively for those who had some real-world experiences related to the domain under study, but who had not reached the proficiency level in the domain of instructional design, as demonstrated by the multiple-case analyses of Kathryn (QG), Simon (QE), and Chris (QG). For example, although Simon was new to the IPT program and was taking the first instructional design course, his think-aloud protocols showed that his problem space was much larger, his understanding of instructional design problem was much deeper and more comprehensive than Jane and Steven, who had been in the graduate program for a year and had taken two ID courses and a few instructional multimedia development courses. A lack of real-world experiences in instructional design made it difficult to activate one's prior knowledge for problem solving in the ill-defined domain of instructional design.

\section{Question Prompts of Different Cognitive Functions for \\ Different Levels of Domain Knowledge and Experience}

For novice learners, the question prompts seemed to play the role of helping them to articulate, elaborate and self-evaluate their thinking process. For example, it was observed that Simon followed the question prompts and articulated his reasoning in response to each of the questions. The question prompts guided him step by step through the problem-solving processes. Jane, who had only some classroom experience with needs assessment, found that the question prompts were helpful in "articulating her thoughts" and "developing her arguments."

However, for the participants with a higher level of prior knowledge and experience, question prompts served as a checklist more than procedural guidance to help them self-monitor and self-reflect their problem-solving processes. For instance, as indicated by Tina in the interview, the question prompts reminded her to look into some of the needs assessment procedures she had previously overlooked in her pre-case study. The question prompts also helped Elaine to think more comprehensively and deeply on the problem being analyzed, and organize her argument more coherently, even though she thought she had a lot of instructional design experience.

\section{DISCUSSION}

The study had three primary goals: a) to confirm the previous findings about the effects of question prompts in scaffolding ill-structured problem solving, particularly in a different domain (i.e., instructional design) and in the Webbased learning environment; b) to investigate the optimal conditions in which question prompts worked most effectively; and c) to examine the interactions between different types of question prompts (QE vs. QG) and various learners' 
variables: learners' prior knowledge, background experience, and problemsolving competence.

The results supported Ge and Land's (2003) findings with respect to the effects of question prompts in scaffolding ill-structured problem solving, indicating that question prompts can also be applied in a complex domain such as instructional design and in Web-based learning environments, especially when learners are novice problem solvers. Overall, the study showed that both QE and QG prompts had beneficial effects in guiding learners through problem-solving processes, elaborating their thinking, and monitoring and evaluating the solution process. One of the examples was that the question prompts helped the participants identify relevant factors and consider alternatives for problem representation, which are some of the skills that need to be developed in novice designers in the process of becoming experts (Perez \& Emery, 1995).

Further, the study indicated that the dual features of the question prompts (i.e., procedural guidance and elaboration) served different cognitive and metacognitive functions at various points of the problem-solving processes in ID tasks. The feature of procedural guidance afforded by the question prompts helped the students to organize information for problem representation, decompose the problem for effective solutions, and break down the problem-solving processes into manageable steps. For more advanced learners, it was observed that procedural guidance also served as a checklist to help them monitor and evaluate their problem-solving processes.

By comparison, the elaboration feature of the question prompts facilitated the students in elaborating the initial state of the problem, identifying constraints, and considering different alternatives for representing problems and constructing problem spaces, which in turn, helped them to consider, compare, and select viable solutions. The elaboration feature also facilitated students to monitor and evaluate their thinking process as a result of cognition and metacognition operating in a correlated manner (Salomon, Globerson, \& Guterman, 1989).

The study revealed the optimal conditions when question prompts worked most effectively, for example: when students had relevant prior knowledge and real-world experience; when they were not led by their assumptions; when students were less competent in solving ill-structured ID problems; when the question prompts were matched with students' levels of problem-solving competence, and when students were facilitated in their problem-solving processes rather than writing processes.

In addition, the study also explored the interactive effects between question prompts and different learner variables, suggesting a need to design question prompts adaptively to meet the needs of individuals with different levels of prior knowledge, real-world experience, and problem-solving competence. As research question 2 and 3 had not been investigated in the previous research, the findings to these two questions have contributed to our knowledge about the nature of question prompts as a scaffolding strategy, especially in supporting 
ill-structured problem solving in Web-based learning environments. The findings raised some issues or implications for further discussion in the space below.

\section{Different Question Prompting Conditions Aligned with Different Levels of Problem-Solving Competence}

Given the two conditions, QE (i.e., in which students were "forced" to provide answers to the question prompts) and QG (i.e., in which students could choose to use the question prompts or skip them), it was expected that the students in the QE condition represented the problem and elaborated thoughts better than the students in the QG condition. However, the study revealed that the resulting effects of the QE or the QG prompts were dependent on the levels of participants' competence in solving an ill-structured problem. This finding disagrees with Zellermayer et al.'s (1991) finding, which indicated that the group who were conditioned to follow procedural guidance performed significantly better in a writing task than the other group, who received the same procedural guidance but had the choice to use it or not. It is evident that different levels of student problem-solving competence interact with different types of question prompts on students' problem-solving performance; therefore, this interactive effect should be taken into consideration when designing question prompts.

The QE prompts seemed to work effectively for Simon, who was just starting his education in ID, and Jane, who had lower competence in solving real-world ID problems, even though she had been studying in the program for a year. However, the QE prompts did not seem to be appropriate for Elaine, who showed the advanced level of competence in solving instructional design problems and stated that the question prompts had interfered with her thought flow. In her case, QG prompts may have worked more effectively, as indicated by Kathryn and Chris' cases, which illustrated that a sufficient level of competence in solving ID problems had enabled them to use QG prompts effectively to guide their problem-solving activities.

The study suggests the need to design question prompts adaptively for individuals with different competence levels in problem solving, especially in the context of Web-based, computer-supported learning environments. For students with lower problem-solving competence in a given content domain, QE prompts should be designed to guide the process, to prompt for explanation, elaboration, and justification. For students with a higher competence level, QG prompts can be provided as a guideline or a checklist to facilitate self-evaluation and selfreflection. Additionally, for less competent students, question prompts can be provided during their problem solving as a scaffold, while for more competent 
students, question prompts can be provided after they have completed problemsolving tasks to facilitate their self-evaluation and self-reflection.

\section{Effects of Prior Knowledge and Real-World Experience}

An important finding of the study is that students' prior knowledge and real-world experience in the content domain have an influence in maximizing the use of question prompts. As observed in Simon's case, although he was a new student in the program of ID, his background experience related to teaching and curriculum design helped him to take advantage of the QE prompts, which effectively facilitated his elaboration of thinking and integration of the new knowledge with the existing knowledge. On the contrary, Steven's case indicated that without a sufficient repertoire of real-world experiences, question prompts had less effect on elaborating his thoughts. Since one of the major functions of question prompts is to activate students' prior knowledge in order to integrate new knowledge, students' prior knowledge in a relevant domain is essential to the learning situation (King, 1992).

The study offered partial explanation to the query regarding student tendencies to ignore question prompts when provided to them. Students might not know how to respond to the question prompts because of their limited problem space as a result of lack of real-world experiences, as was demonstrated by Steven's case. It is clear that prior knowledge gained from the classroom alone is insufficient in helping students represent complex, ill-defined problems. Hence, additional scaffolds are necessary in order to expand students' schema, broaden their problem space, and maximize the positive effects of question prompts. One of the strategies is to expose students to real-world experiences at an early stage through various opportunities, including case studies, real-world projects, practicum, and internships. These opportunities may help students develop structural knowledge, which is an essential knowledge component used to develop procedural knowledge for solving ill-structured, domain-specific problems (Jonassen, Beissner, \& Yacci, 1993).

\section{Mindful Use of Question Prompts and Alternative Scaffolds}

The overlook of question prompts by some students might also be due to students' self-perception of their problem-solving skills. Some students thought they already knew how to think and solve problems. Consequently, they did not perceive question prompts as a helpful or necessary support, even though they had not reached the desired level of competence in solving ill-structured problems. They either chose to respond to some of the questions, as in Chris' case, or overlooked the questions completely. This situation is more likely to happen in 
the context where students can use question prompts on a voluntary basis, as in the QG condition.

Apart from the other factors (e.g., learner's prior knowledge, real-world experiences, and problem-solving competence), intentional use of question prompts also determines the effectiveness of question prompts to a large extent (Zellermayer et al., 1991). Kathryn's case illustrated this point. Kathryn was observed to be deliberately using the QG prompts to guide her problem-solving processes; as a result she benefited a great deal from the question prompts. Therefore, it is important to make students aware of the function and the usefulness of question prompts and encourage them to use the cognitive tool provided to them voluntarily.

Alternatively, for novice learners it may be necessary to provide them with QE prompts to ensure that they do not skip some important components or processes during problem solving. The QE prompts may be gradually withdrawn and replaced by QG prompts as students grow more competent in problem solving. Some other kinds of scaffolds may also be beneficial to accommodate different individuals' needs in the Web-based learning environment, for instance, presenting expert examples for modeling and reflection, and combining question prompts with human tutor feedback (Azevedo et al., 2004; van den Boom et al., 2004) via e-mails or other online communication media.

\section{Different Scaffolds for Different Cognitive Tasks: Problem-Solving Processes vs. Written Products}

An unexpected finding of this study was that the reasoning process of problem solving and the productive process of writing the solution report seemed to be two separate cognitive tasks, which were not necessarily compatible with one another. This finding has two implications. First, it leads us to question the sufficiency of using written reports as a measurement for students' problem-solving abilities in terms of cognitive and metacognitive processes. It suggests that supplemental instruments are necessary to measure students' problem-solving performance accurately.

Second, the study indicates a need for developing scaffolds focusing on the productive process of writing solution reports. Scardamalia et al. (1984) identified two problem spaces in terms of writing tasks: content space for idea production and rhetorical space for text production, and argued that it was important to help students make connections between the two spaces through reflective thinking processes. In the present study, the scaffolds only focused on facilitating problem-solving processes, but they had not been designed to facilitate students' productive process involving writing or verbalizing a solution report. Thus, we argue that for future research, the productive or writing prompts need to be designed to help students organize information they have elaborated or generated for problem representation, link solutions to problem representation, and support 
solution with evidence. Showing students good examples of solution reports may also be beneficial in helping students understand the expectations and the requirements for problem-solving reports.

\section{CONCLUSIONS}

The findings of the present study have particularly important and meaningful implications to the design of Web-based instruction, which has become an increasingly popular and convenient instructional delivery system. It has been recognized that educators who conduct online courses often become overwhelmed by the number of e-mails they receive from students and the amount of the feedback they have to provide (Kauffman, 2004). Kauffman argued that question prompting was a powerful strategy that could significantly reduce teachers' workload while providing efficient, effective, and automatic feedback to help students self-monitor their learning process.

Furthermore, we argue that the question prompts investigated in the present study were designed to provide just-in-time support to students as they were engaged in complex, ill-structured problem-solving tasks independently. While some other studies (e.g., Azevedo et al., 2004; van den Boom et al., 2004) reported that dynamic scaffolding, such as a human tutor, was more effective than static scaffolding, this study was intended to address a situation when no human scaffolding was immediately available. In that regard, it becomes a critical issue how we can design question prompts adaptively and map the right type of question prompts to different learners in order to maximize the benefits of question prompts.

Although this study was limited in its sample size, the comparative, multiplecase study method allowed us to gather rich data of various sources and conduct an in-depth analysis of the effects of question prompts in scaffolding illstructured problem-solving processes under different prompting conditions for participants of different levels of prior knowledge and experience. Further research is necessary to investigate the similar research questions by conducting experimental studies on a larger sample size. Future study should also investigate the different effects between procedural prompts, which are provided during students' problem solving and aim at providing procedural facilitation; and reflective prompts, which are provided after students' problem solving and designed to encourage reflection and revisions of their problemsolving processes. It is also worth comparing the effects of question prompts with the effects of other types of instructional scaffolds in supporting illstructured problem solving. Finally, investigation should also be carried out to examine the transfer effects of the question prompts in helping students to internalize the problem-solving approach they have gained from question prompt modeling. 


\section{APPENDIX A Sample Case Study}

\section{Case Study 1}

Professor Lou teaches English literature at College of Arts and Sciences. Literature is a subject that many students are not really interested in but have to take for credits or other reasons. In order to get her students interested in her class, she has come up with lots of innovative ideas to stimulate students' interest in classical literature, including the use of Internet. Recently she has received a small grant for integrating technology in instruction. She was very excited about it and wanted to implement Web-based instruction to facilitate her classroom teaching. She has asked you, a student intern with the Faculty Technology Support Center, to develop a Web-based course for her. She mentioned several advantages of using technology to deliver instruction: first, the Web can provide students with various resources, such as Shakespeare's time and historical background; second, the Web can provide students with easy access to various visuals, such as pictures, audio, and video clips, which students can study any time of the day.

You are very excited about the prospective design project and would like to start working on it right away, as you could play to the strength of your Web development skills. At the same time, however, you feel that you have not obtained sufficient information that would legitimately allow you to start the design.

Your task is to work with Professor Lou to (a) identify the problem(s); (b) develop a formal needs assessment plan; and (c) write your rationale to justify your plan.

\section{APPENDIX B Sample Question Prompts}

\section{Question Prompts for Case Study 1}

1. Is this design project in response to a problem or a need? Is there really a need for Web-based instruction? How do you know? How can you determine it?

2. What might be the major causes for students' low motivation in learning classical literature? Do you think Web-based instruction can be a solution? Why or why not? How do you think the students may respond to such a solution?

3. Do you think that the information provided by Professor Lou is sufficient for you to design the Web-based instructional modules? Why or Why not?

4. What information do you need to find out before investing your time and effort into the development of the Web-based instruction?

5. What specific questions should be asked in order to find out the gap/ discrepancy between the optimal and the actual situations in the following areas: the learning environment, learner characteristics, learning goals, contextual factors, etc.? 
6.1. From what types of people are you going to gather the information to answer the questions you generated in \#5 above? Why?

6.2 What kind of investigation techniques are you going to use?

6.3. Why do you suggest using each of the techniques above? Discuss advantages and disadvantages of each of the techniques.

\section{REFERENCES}

Azevedo, R., Cromley, J. G., \& Seibert, D. (2004). Does adaptive scaffolding facilitate students' ability to regulate their learning with hypermedia? Contemporary Educational Psychology, 29, 344-370.

Chi, M., Bassok, M., Lewis, M., Reimann, P., \& Glaser, R. (1989). self-explanations: How students study and use examples in learning to solve problems. Cognitive Science, $13,145-182$.

Chi, M. T. H., \& Glaser, R. (1985). Problem solving ability. In R. J. Sternberg (Ed.), Human abilities: An information processing approach (pp. 227-250). New York: W. H. Freeman and Company.

Cognition and Technology Group at Vanderbilt. (1990). Anchored instruction and its relationship to situated cognition. Educational Researcher, 19, 2-10.

Cognition and Technology Group at Vanderbilt. (1993). Designing learning environments that support thinking: The Jasper series as a case study. In T. M. Duffy, J. Lowyck, \& D. H. Jonassen (Eds.), Designing environments for constructive learning (pp. 9-36). Berlin: Springer-Verlag.

Davis, E. A., \& Linn, M. (2000). Scaffolding students' knowledge integration: Prompts for reflection in KIE. International Journal of Science Education, 22(8), 819-837.

Dick, W. O., Carey, L., \& Carey, J. O. (2001). The systematic design of instruction (5th ed.). NY: Longman.

Dijkstra, S. (2001). The design space for solving instructional-design problems. Instructional Science, 29, 275-290.

Ertmer, P. A., \& Newby, T. J. (1996). The expert learner: Strategic, self-regulated, and reflective. Instructional Science, 24, 1-24.

Feltovich, P. J., Spiro, R. J., \& Coulson, R. L. (1989). The nature of conceptual understanding in biomedicine: The deep structure of complex ideas and the development of misconceptions. In D. A. Evans \& V. L. Patel (Eds.), Cognitive science in medicine: Biochemical modeling (pp. 111-172). Cambridge, MA: MIT Press.

Feltovich, P. J., Spiro, R. J., Coulson, R. L., \& Feltovich, J. (1996). Collaboration within and among minds: Mastering complexity, individually and in groups. In T. Koschmann (Ed.), CSCL: Theory and practice of an emerging paradigm (pp. 25-44). Mahwah, NJ: Lawrence Erlbaum Associates.

Ge, X., \& Land, S. M. (2003). Scaffolding students' problem-solving processes in an ill-structured task using question prompts and peer interactions. Educational Technology Research and Development, 51(1), 21-38.

Ge, X., \& Land, S. M. (2004). A conceptual framework of scaffolding ill-structured problem solving processes using question prompts and peer interactions. Educational Technology Research and Development, 52(2), 5-22. 
Gick, M. L. (1986). Problem solving strategies. Educational Psychologist, 21(1\&2), 99-120.

Gick, M. L., \& Holyoak, K. J. (1980). Analogical problem solving. Cognitive Psychology, 12, 306-355.

Greene, B. A., \& Land, S. M. (2000). A qualitative analysis of scaffolding use in a resource-based learning environment involving the world wide web. Journal of Educational Computing Research, 23(2), 151-180.

Gustafson, K. L., \& Branch, R. M. (2002). What is instructional design? In R. A. Reiser \& J. V. Dempsey (Eds.), Trends and issues in instructional design and technology (pp. 16-25). New York: Merrill.

Jonassen, D. H. (1997). Instructional design models for well-structured and ill-structured problem-solving learning outcomes. Educational Technology Research and Development, 45(1), 65-94.

Jonassen, D. H. (1999a). Designing constructivist learning environments. In C. M. Reigeluth (Ed.), Instructional design theories and models: Vol. II. A new paradigm of instructional theory, (pp. 215-239). Mahwah, NJ: Lawrence Erlbaum Associates.

Jonassen, D. (1999b). Forward. In P. A. Ertmer \& J. Quinn (Eds.), The ID CaseBook: Case studies in instructional design (pp. v-vi). Upper Saddle River, NJ: Prentice-Hall.

Jonassen, D. H., Beissner, K., \& Yacci, M. (1993). Structural knowledge. Hillsdale, NJ: Lawrence Erlbaum Associates.

Kauffman, D. F. (2004). Self-regulated learning in Web-based environments: Instructional tools designed to facilitate cognitive strategy use, metacognitive processing, and motivational beliefs. Journal of Educational Computing Research, 30(1\&2), 139-161.

King, A. (1991). Effects of training in strategic questioning on children's problem-solving performance. Journal of Educational Psychology, 83(3), 307-317.

King, A. (1992). Facilitating elaborative learning through guided student-generated questioning. Educational Psychologist, 27(1), 111-126.

King, A. (12994). Guiding knowledge construction in the classroom: Effects of teaching children how to question and how to explain. American Educational Research Journal, 31(2), 338-368.

Kitchener, K. S. (1983). Cognition, metacognition, and epistemistic cognition: A threelevel model of cognitive processing. Human Development, 26, 222-232.

Kluwe, R. H., \& Friedrichsen, G. (1985). Mechanism of control and regulation in problem solving. In J. Kuhl \& J. Beckmann (Eds.), Action control: From cognition to behavior. New York: Springer-Verlag.

Koschmann, T., Kelson, A. C., Feltovich, P. J., \& Barrows, H. S. (1996). Computersupported problem-based learning: A principled approach to the use of computers in collaborative learning. In T. Koschmann (Ed.), CSCL: Theory and practice of an emerging paradigm (pp. 83-124). Mahwah, NJ: Lawrence Erlbaum Associates.

Lin, X., \& Lehman, J. D. (1999). Supporting learning of variable control in a computer-based biology environment: Effects of prompting college students to reflect on their own thinking. Journal of Research in Science Teaching, 36(7), 837-848.

Miles, M. B., \& Huberman, A. M. (Eds.). (1994). Qualitative data analysis: An expanded sourcebook (2nd ed.). Thousand Oaks, CA: Sage Publications. 
Palincsar, A. S., \& Brown, A. L. (1984). Reciprocal teaching of comprehensionfostering and comprehension-monitoring activities. Cognition and Instruction, 2, 117-175.

Perez, R. S., \& Emery, C. D. (1995). Designer thinking: How novices and experts think about instructional design. Performance Improvement Quarterly, 8(3), 80-95.

Reigeluth, C. M. (1999). What is instructional-design theory and how is it changing? In C. M. Reigeluth (Ed.), Instructional-design theories and models: Vol. II. A new paradigm of instructional theory (pp. 5-29). Mahwah, NJ: Lawrence Erlbaum Associates.

Rosenshine, B., \& Meister, C. (1992). The use of scaffolds for teaching higher-level cognitive strategies. Educational Leadership, 4, 26-33.

Rosenshine, B., Meister, C., \& Chapman, S. (1996). Teaching students to generate questions: A review of the intervention studies. Review of Educational Research, 66(2), 181-221.

Salomon, G., Globerson, T., \& Guterman, E. (1989). The computer as a zone of proximal development: Internalizing reading-related metacognitions from a reading partner. Journal of Educational Psychology, 81(4), 620-627.

Scardamalia, M., \& Bereiter, C. (1985). Fostering the development of self-regulation in children's knowledge processing. In S. F. Chipman, J. W. Segal, \& R. Glaser (Eds.), thinking and learning skills: Research and open questions (Vol. 2, pp. 563-577). Hillsdale, NJ: Lawrence Erlbaum Associates.

Scardamalia, M., Bereiter, C., \& Steinbach, R. (1984). Teachability of reflective processes in written composition. Cognitive Science, 8(2), 173-190.

Shoenfeld, A. H. (1985). Mathematical problem-solving. San Diego, CA: Academic Press.

Sinnott, J. D. (1989). A model for solution of ill-structured problems: Implications for everyday and abstract problem solving. In J. D. Sinnott (Ed.), Everyday problem solving: Theory and application (pp. 72-99). New York: Praeger.

Stake, R. E. (2000). Case studies. In N. K. Denzin \& Y. S. Lincoln (Eds.), Handbook of qualitative research (pp. 435-454). Thousand Oaks, CA: Sage Publications.

van den Boom, G., Paas, F., van Merriënboer, J. J. G., \& van Gog, T. (2004). Reflection prompts and tutor feedback in a web-based learning environment: Effects of students' self-regulated learning competence. Computers in Human Behavior, 20, 551-567.

van Merriënboer, J. J. G., Kirschner, P. A., \& Kester, L. (2003). Taking the load off a learner's mind: Instructional design for complex learning. Educational Psychologist, 38(1), 5-13.

Voss, J. F., \& Post, T. A. (1988). On the solving of ill-structured problems. In M. H. Chi, R. Glaser, \& M. J. Farr (Eds.), The nature of expertise (pp. 261-285). Hillsdale, NJ: Lawrence Erlbaum Associates.

Voss, J. F., Wolfe, C. R,, Lawrence, J. A., \& Engle, R. A. (1991). From representation to decision: An analysis of problem solving in international relations. In R. J. Sternberg \& P. A. Frensch (Eds.), Complex problem solving: Principles and mechanisms. Hillsdale, NJ: Lawrence Erlbaum Associates.

Yin, R. K. (2002). Case study research: Design and methods (3rd ed.). Thousand Oaks, CA: Sage Publications. 


\section{8 / GE, CHEN AND DAVIS}

Zellermayer, M., Salomon, G., Globerson, T., \& Givon, H. (1991). Enhancing writingrelated metacognitions through a computerized writing partner. American Educational Research Journal, 28(2), 373-391.

Direct reprint requests to:

Dr. Xun Ge

Department of Educational Psychology

The University of Oklahoma

820 Van Vleet Oval

321 Collings Hall

Norman, OK 73019-2041

e-mail: xge@ou.edu 